\title{
An Energy-Efficient, Dynamic Voltage Scaling Neural Stimulator for a Proprioceptive Prosthesis
}

\author{
Ian Williams, Student Member, IEEE, and Timothy G. Constandinou, Senior Member, IEEE
}

\begin{abstract}
This paper presents an 8 channel energy-efficient neural stimulator for generating charge-balanced asymmetric pulses. Power consumption is reduced by implementing a fullyintegrated DC-DC converter that uses a reconfigurable switched capacitor topology to provide 4 output voltages for Dynamic Voltage Scaling (DVS). DC conversion efficiencies of up to $82 \%$ are achieved using integrated capacitances of under $1 \mathrm{nF}$ and the DVS approach offers power savings of up to $50 \%$ compared to the front end of a typical current controlled neural stimulator. A novel charge balancing method is implemented which has a low level of accuracy on a single pulse and a much higher accuracy over a series of pulses. The method used is robust to process and component variation and does not require any initial or ongoing calibration. Measured results indicate that the charge imbalance is typically between $0.05 \%-0.15 \%$ of charge injected for a series of pulses. Ex-vivo experiments demonstrate the viability in using this circuit for neural activation. The circuit has been implemented in a commercially-available $0.18 \mu \mathrm{m} \mathrm{HV}$ CMOS technology and occupies a core die area of approximately $2.8 \mathrm{~mm}^{2}$ for an 8 channel implementation.
\end{abstract}

Index Terms-Neural stimulator, current mode, Dynamic Voltage Scaling, power efficient, charge balancing, proprioception.

\section{INTRODUCTION}

I $\mathrm{N}$ recent years there have been exciting demonstrations of the potential benefits offered by electrical neural stimulators in a wide variety of applications such as vision and vestibular prostheses [1]-[4]. Cochlear implants remain the main commercial success to date [5]-[7], but there are a wealth of sensory and motor rehabilitation applications that are showing rapid progress.

Despite the breadth of applications, the fundamental aim of all neural stimulators is the same - to deliver a packet of charge to an area of neural tissue and to thereby initiate an action potential. In practice safe stimulation means that the packet of charge delivered to the tissue also needs to be removed - giving a charge balanced stimulation. Unbalanced stimulations give rise to DC currents flowing across the electrode / tissue interface and have been linked with tissue damage and deterioration of the electrode [8].

Delivery and recovery of this charge packet is typically achieved using a biphasic voltage or current controlled waveform. The former is much more power efficient but does not allow the amount of charge delivered to be controlled. This has safety implications and also means that more frequent recalibration of stimulation intensity is required; as such

All authors are with the Centre for Bio-Inspired Technology and the Department of Electrical and Electronic Engineering, Imperial College London, SW7 2BT, UK e-mail: \{i.williams10, t.constandinou\}@imperial.ac.uk

Manuscript received; revised current control is commonly preferred. However, the high degree of power wasted in current control (see Section III) is a serious concern in implanted systems for two reasons: (1) the power consumed is ultimately dissipated thermally and may damage the surrounding tissue; (2) the increased energy capacity requirements reduce primary battery lifetime and increase the number of charging cycles for a secondary battery.

This paper presents a low power neural stimulator targeted at a Peripheral Nervous System (PNS) implant for providing proprioceptive feedback from a prosthetic limb. Preliminary work towards this has been reported in [9]. This paper details the complete integrated circuit implementation and provides experimental results demonstrating both chip functionality and ex-vivo viability.

The paper is organised as follows: Section II introduces the application; Section III introduces the system concept; Section IV details the circuit implementation; Section V presents simulated and measured results; and Section VI draws the conclusion.

\section{Proprioceptive Prosthesis}

Proprioception refers to the body's ability to sense the position and motion of the various parts of the body and the forces that are being exerted by it. It is a sense that we are often not aware of, and yet it is a crucial feedback mechanism for skilled motor control and learning new motor tasks [10][12].

There are millions of people worldwide who suffer from impaired proprioception either because of neural damage (such as sensory neuropathy caused by diabetes [13], [14]), or because they use an artificial limb. Broadly speaking any of these

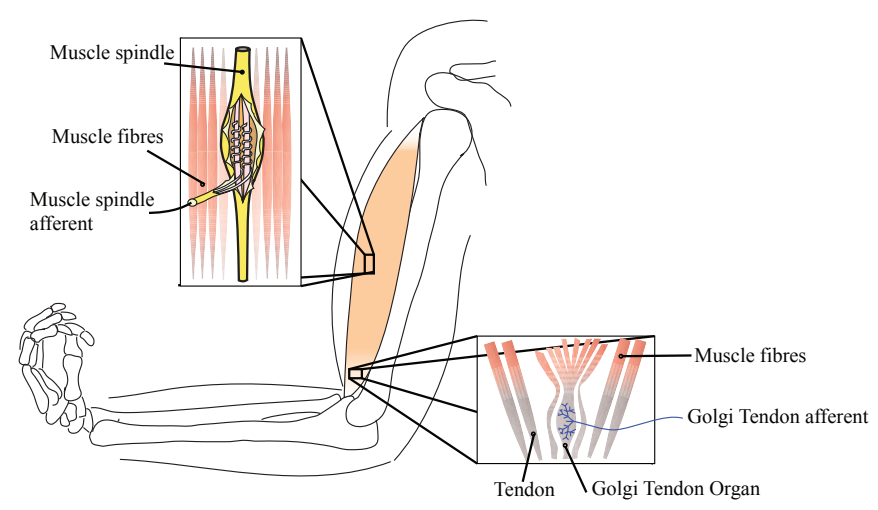

Fig. 1. Key nerve receptors for proprioception: a muscle spindle and a Golgi Tendon Organ (GTO). 


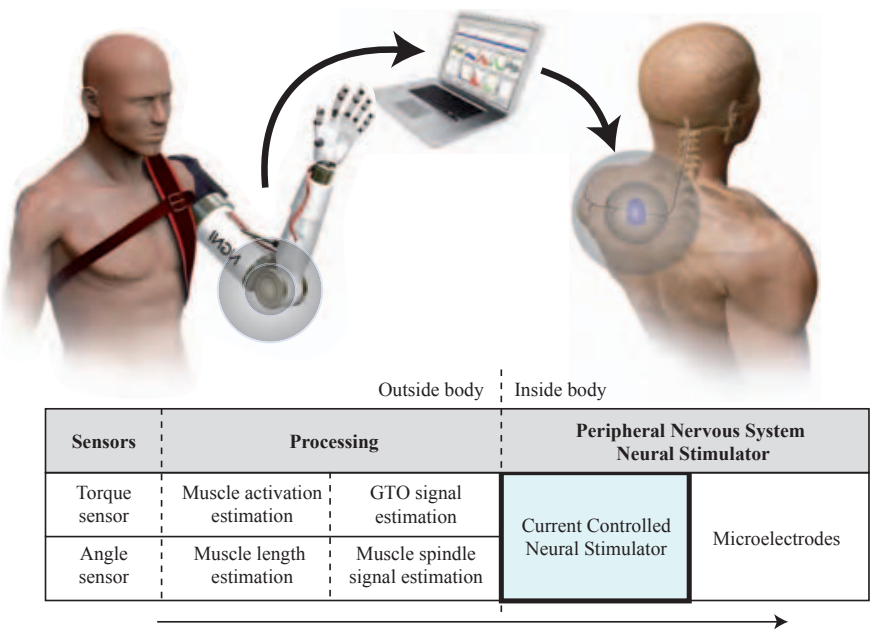

Fig. 2. Top level overview of the proposed prosthesis showing the different functional blocks. Arrow indicates the direction of data flow through the system.

people could benefit from a proprioceptive prosthesis, provided they have a degree of motor control in the impaired limb(s) and an intact proprioceptive system proximal to the implant site. However, on the grounds of feasibility and perceived user benefit, our work initially focuses on those people who use artificial limbs and in particular upper arm amputees.

In recent years highly capable powered prostheses such as the DEKA arm have been created, with the tantalizing potential to provide amputees with an artificial arm of comparable dexterity, size and weight to the human arm [15]. However, enabling the user to effectively control an arm of this complexity remains a major challenge. A significant amount of research has been undertaken to develop improved interfaces for prosthesis control and techniques such as Targeted Muscle Reinnervation have already been shown to provide major benefits [16], [17]. However, nearly all of this research has focused on feed forward open loop control techniques which limits the ultimate performance that can be achieved and also means that the user must visually monitor their prosthetic limb when using it [18].

Our research aims to close the loop on these control techniques by using software to model the movements of a prosthetic limb and electrical neural stimulation to create a feedback path that provides proprioceptive information to the user. We will initially be focussing on flexion and extension of the elbow joint and mimicking the signals provided by 2 key nerve receptors for proprioception: (1) muscle spindles which lie within a muscle and provide feedback on the length and rate of stretch of that muscle; and (2) Golgi Tendon Organs (GTOs) which are located at the boundary between muscles and tendons and measure the amount of force a muscle is exerting [10]-[12]. These receptors are illustrated in Fig. 1.

The concept for this system is shown in Fig. 2 and essentially consists of three main blocks. The first part consists of sensors to monitor the movement and joint torques of the prosthetic arm. Secondly a processing block uses biomechanical models to estimate the lengths of various muscles and the muscle activations that, in a healthy arm, would best fit

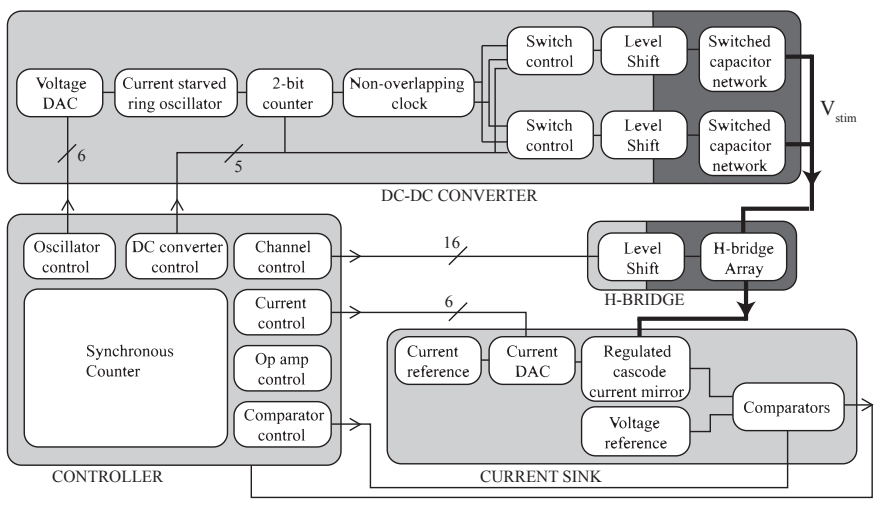

Fig. 3. Overview of the proposed neural stimulator. Light grey areas are powered from $1.8 \mathrm{~V}$ supply, dark grey areas from a $6 \mathrm{~V}$ supply. Thick black lines indicates the main current path.

the sensor data. This processing block then uses models of the neural firing patterns of muscle spindles and GTOs to generate a stimulation pattern that is passed onto the third and final system block - the neural stimulator which stimulates axons in the Peripheral Nervous System proximal to the point of impairment. The design of a neural stimulator for a proprioceptive prosthesis is the subject of this paper.

\section{SySTEM OVERVIEW}

The neural stimulator presented here is based on Current Controlled Stimulation (CCS) but makes use of efficient DCDC conversion to address the primary issue with CCS - namely the excessive power consumption. Section III-A provides a description of the main sources of power waste and how the DC-DC conversion is used to mitigate this. The stimulator is further enhanced by a novel charge balancing approach (discussed in Section III-B), which is included to improve the performance as well as the safety of the stimulation without requiring external components (e.g. off-chip discrete capacitors or inductors). The fully integrated nature of this design enables miniaturisation of the implant and helps reduce the number of fragile bonding wires required.

The main system components are shown in Fig. 3. At a top level it consists of: a Digital Control block which outputs timing and control signals; a DC-DC converter which provides a variable stimulation voltage; an H-bridge array which selects the electrodes involved in each stimulation; and finally a Current Sink which controls the amount of current that flows through the electrodes during a stimulation. A second DC-DC converter has additionally been included to provide the $1.8 \mathrm{~V}$ supply to power the system core.

\section{A. Energy Efficiency}

Power consumption in current-mode neural stimulation is typically dominated by the power used in the front-end to drive the current flow (i.e. charge stimulus) into and out of electrodes (see Fig. 5(a)). Kelly in [19] calculated that, even using a low power current source, as much as $92 \%$ of the front end power is dissipated as heat by the current controlling transistors. The reason for this can be observed by looking 


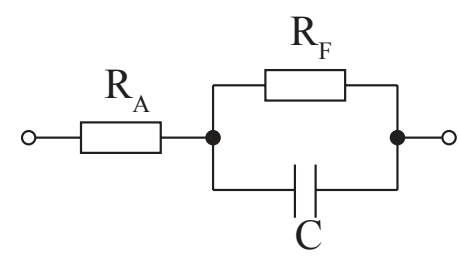

Fig. 4. Lumped circuit model of the electrode-tissue interface.

at the electrodes, voltages, and currents associated with this proprioceptive stimulator.

For our target application, we believe that sub-fascicular level selectivity will be required and as such we are expecting to use micro-electrode arrays or intra-fascicular devices such as the TIME electrodes [20]. These electrodes are usually modelled as a resistor in series with a parallel resistor and capacitor (see Fig. 4). This parallel resistance $\left(R_{F}\right)$ corresponds to the Faradaic flow of current into the tissue due to nonreversible redox reactions at the interface. $R_{F}$ is typically very large and so for the simplicity of the analysis in this paper it will be assumed to be infinite. Based on TIME electrode impedances we are therefore assuming a resistor $(7 \mathrm{k} \Omega)$ in series with a capacitor $(7 \mathrm{nF})$ as our electrode model for this analysis. In practice the impedance of the electrodes and tissue will vary considerably and the impact of this on efficiency will be briefly discussed in Section VI.

Selective stimulation of efferent neurons (similar to the afferent Type Ia and Ib neurons we are targeting) in the human PNS has been demonstrated in [21], [22] to occur with charge packets of between $11 \pm 5 \mathrm{nC}$ to $29 \pm 17 \mathrm{nC}$. Given these values our stimulator will be designed to deliver up to $50 \mathrm{nC}$ in a $100 \mu$ s pulse (a common pulse duration). The voltage compliance of the system needs to be greater than the voltage developed across the electrodes given by:

$$
V_{e}=I_{\text {stim }} \times R_{\text {elec }}+\frac{Q_{\text {total }}}{C}
$$

where $V_{e}, I_{\text {stim }}, R_{\text {elec }}, Q_{\text {total }}$ and $C$ are the voltage across the electrodes, stimulation current, electrode resistance, charge stimulus and capacitance respectively. Using our chosen impedance and charge packet values, this means our system requires a voltage compliance of $\geq 10.64 \mathrm{~V}$. However, it should be noted that this is just the peak voltage and for the vast majority of the time the system does not need to operate at this voltage (see Fig. 5(b)). A typical neural stimulator, however, has fixed supplies and as such there is no option. This excess voltage leads to waste power (see Fig. 5(c)).

Ideally the voltage of the power supply to the front end current control would match the solid black line in Fig. 5(c) and, as demonstrated by Arfin et al. [23], a switching DC-DC converter can be used to approximate this. However, a switching DC-DC converter requires bulky external components which we are looking to avoid and has not yet been shown to be compatible with our desire for accurate charge balancing. In this paper we discuss the use of a fully integrated Switched Capacitor DC-DC converter that creates a series of discrete output voltages as indicated in Fig. 5(d) in order to achieve high voltage compliance [24], but also reducing the excess

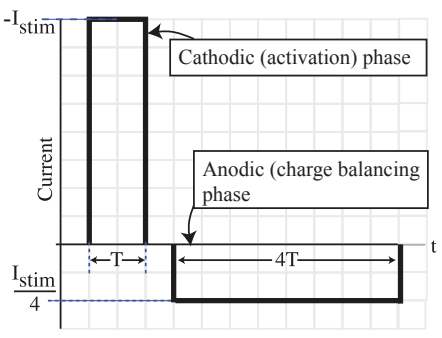

(a)

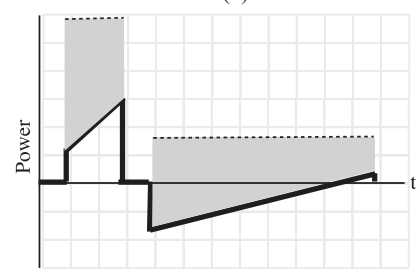

(c)

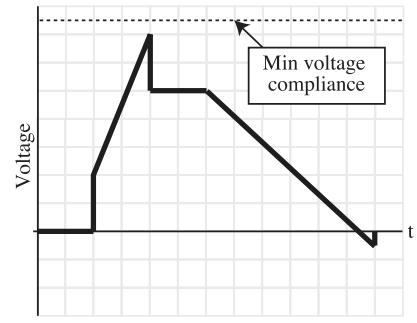

(b)

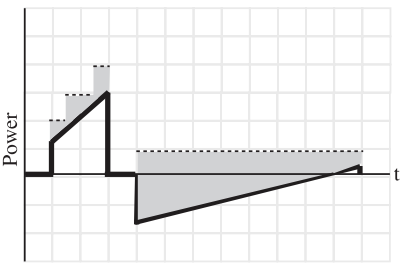

(d)
Fig. 5. Electrical neural stimulation waveforms showing (a) a typical current asymmetric waveform, (b) the voltage across the electrode, (c) the power consumption in a typical (current-mode) stimulator, and (d) power consumption for the proposed scheme. In (c) and (d) the dark line is the theoretical minimum power consumption, dotted line is the actual power consumption and the shaded area is the power wasted.

voltage across current controlling transistors and therefore reducing power consumption. This approach of having a series of discrete voltage steps available on a chip is used widely in low power CPUs and is commonly referred to as Dynamic Voltage Scaling [25].

\section{B. Charge Balancing}

Charge balancing in current controlled stimulation, is fundamentally a challenge of matching the current source in the cathodic phase with the current sink in the anodic phase. One solution to this is to use a calibration phase to match a current sink and source prior to stimulation [26], [27]. However, a more elegant solution is to use an H-bridge, which enables the same current source to be used for both phases (thus eliminating mismatch concerns). The direction of current flow through the electrode is simply switched / steered as shown in Fig. 6(a).
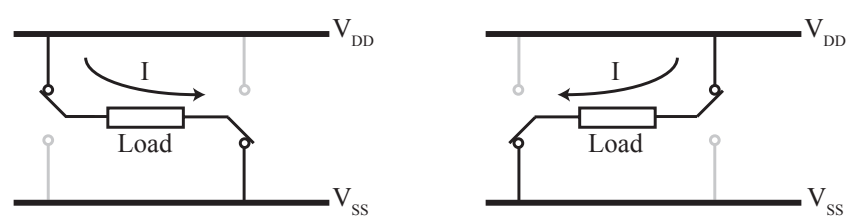

Fig. 6. H-bridge configuration for biphasic current control. Switches allow bi-directional flow of current through a load.

Both these solutions assume that a symmetrical biphasic waveform is used, i.e. the amount of current flowing in the anodic and cathodic phases is the same. However, an asymmetric waveform (Fig. 5(a)) with delay between the cathodic and anodic phases has been shown to provide major benefits such as reducing power consumption and initiating action potentials at significantly lower charge thresholds [28] which improves stimulation safety. 
In this paper we propose a variant on the $\mathrm{H}$-bridge approach that is compatible with an asymmetric waveform and is based on the assumption that it is acceptable to achieve accurate charge balancing over a series of pulses rather than on a single pulse. The fundamental principle is that during the high amplitude cathodic phase $N$ current sinks are acting in parallel to pull current through the electrodes for a time $T$. Then during the low amplitude anodic phase 1 current sink is acting to pull current through the electrodes (in the opposite direction) for a time $N T$. On a single stimulation this will not give accurate charge balancing as the current sinks are not perfectly matched. However, if the current sink that is active in the anodic phase is changed after each stimulation then after $N$ stimulations each sink will have been active for the same amount of time during cathodic and anodic phases giving accurate charge balancing. The value of $N$ can be any real, positive integer and choosing a value for $N$ depends on a tradeoff between the aforementioned benefits of asymmetric pulses versus the increased duration of the anodic phase that occurs as $N$ is increased.

\section{CiRCUit ImPLEMENTATION}

The circuit has been implemented in a commercially available High Voltage $0.18 \mu \mathrm{m}$ CMOS technology provided by AMS / IBM (H18A4/7HV). This section details the transistor level circuit implementation.

\section{A. Controller}

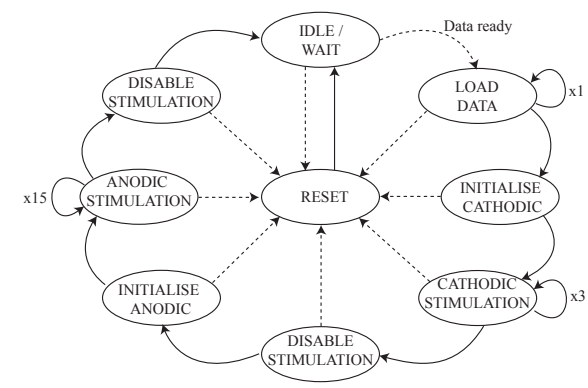

Fig. 7. State diagram for the neural stimulator controller. The solid lines indicate normal clock cycle based transitions, dotted lines indicate transitions caused by data ready (labelled) or reset signals.

This is essentially a finite state machine made up of a synchronous counter and a series of supporting digital logic blocks for controlling the various sub-systems. The stimulus is initiated on receipt of a data ready signal which enables the synchronous counter and reads in a 15-bit instruction that controls the stimulus output. This 15 instruction consists of: channel selection (3-bit), stimulation current (6-bit) and oscillator frequency (6-bit) for the DC-DC converter. The counter runs on a fixed $40 \mathrm{kHz}$ clock and steps through the various states indicated in Fig. 7 to provide the stimulation timing signals and power enable signals to the sub-systems (reducing power consumption) as described in Table I. A single stimulation takes 26 clock cycles $(650 \mu \mathrm{s})$ and therefore this system is currently capable of in excess of 1500 stimulations per second.
TABLE I

CONTROLLER STATE DESCRIPTIONS.

\begin{tabular}{|c|c|c|}
\hline State & $\begin{array}{l}\text { Clock } \\
\text { Cycles }\end{array}$ & Action \\
\hline Idle / wait & N/A & Await data ready signal \\
\hline Load data & 1 & Read in 15 bit control instruction \\
\hline Initialise Cathodic & 2 & $\begin{array}{l}\text { Set \& enable oscillator, current sink and } \\
\text { comparators. }\end{array}$ \\
\hline Cathodic stimulation & 4 & H-bridge set to chosen channel \\
\hline Disable stimulation & 1 & Disable all subsystems \\
\hline Initialise Anodic & 1 & $\begin{array}{l}\text { Set and enable oscillator, current sink } \\
\text { and comparators. }\end{array}$ \\
\hline Anodic Stimulation & 16 & H-bridge set to chosen channel \\
\hline Disable stimulation & 1 & Disable all subsystems \\
\hline Reset & 1 & $\begin{array}{l}\text { Disable all outputs, reset all latches and } \\
\text { flip flops }\end{array}$ \\
\hline
\end{tabular}

\section{B. $D C-D C$ converter}

At the core of this system is a DC-DC converter to provide $V_{\text {stim }}$. This converter is a rapidly reconfigurable switchedcapacitor network capable of generating: $3 \mathrm{~V}, 9 \mathrm{~V}$ and $12 \mathrm{~V}$ from an input power supply of $6 \mathrm{~V}$. These outputs are unregulated and thus drop slightly under load. This, however, does not affect the stimulation output as the current is controlled using a floating source voltage that is independent of the DC-DC converter output. The DC-DC converter operates in a free running mode, i.e. it starts outputting at $3 \mathrm{~V}$ and asynchronously increases the output voltage during a stimulation. The signal for increasing the output voltage is determined by comparing the gate voltages $\left(V_{1}-V_{4}\right.$ in Fig. 10) on the high voltage thin oxide cascode transistors with a reference voltage. When this reference voltage is exceeded (indicating that the cascode transistors are close to leaving the saturation region), a feedback signal is sent to the DC-DC converter to increase the output voltage.

The building blocks of the DC-DC converter are shown in Fig. 8a-e. The first section includes a 6-bit charge scaling DAC which is combined with a current starved ring oscillator to create a digitally controlled oscillator (as shown in Fig. 8a) with an output frequency range of between $50 \mathrm{kHz}$ and 3.3 MHz. Due to the constant current nature of the load, this frequency is linearly related to how much current the converter can supply (for a given voltage ripple on the output). This frequency is therefore tuned according to the current required during the cathodic (high amplitude) phase and the amount of voltage ripple that is acceptable on the output. To prevent excess power consumption during the lower amplitude anodic phase (i.e. when the stimulation current to be delivered is a quarter of the cathodic level) a frequency divider (based on a 2 bit counter) is used.

The output of the oscillator is then passed to a nonoverlapping clock generator (as described in [29]) which generates 2 complementary and non-overlapping clocks at the oscillator frequency. The separation between the two clock phases $\left(C_{1}, C_{2}\right)$ has been designed to be approximately $8 \mathrm{~ns}$ to ensure that, even with process variation and delay mismatch, the complementary switch groups will never be active at the same time (as verified in Monte Carlo and transient 

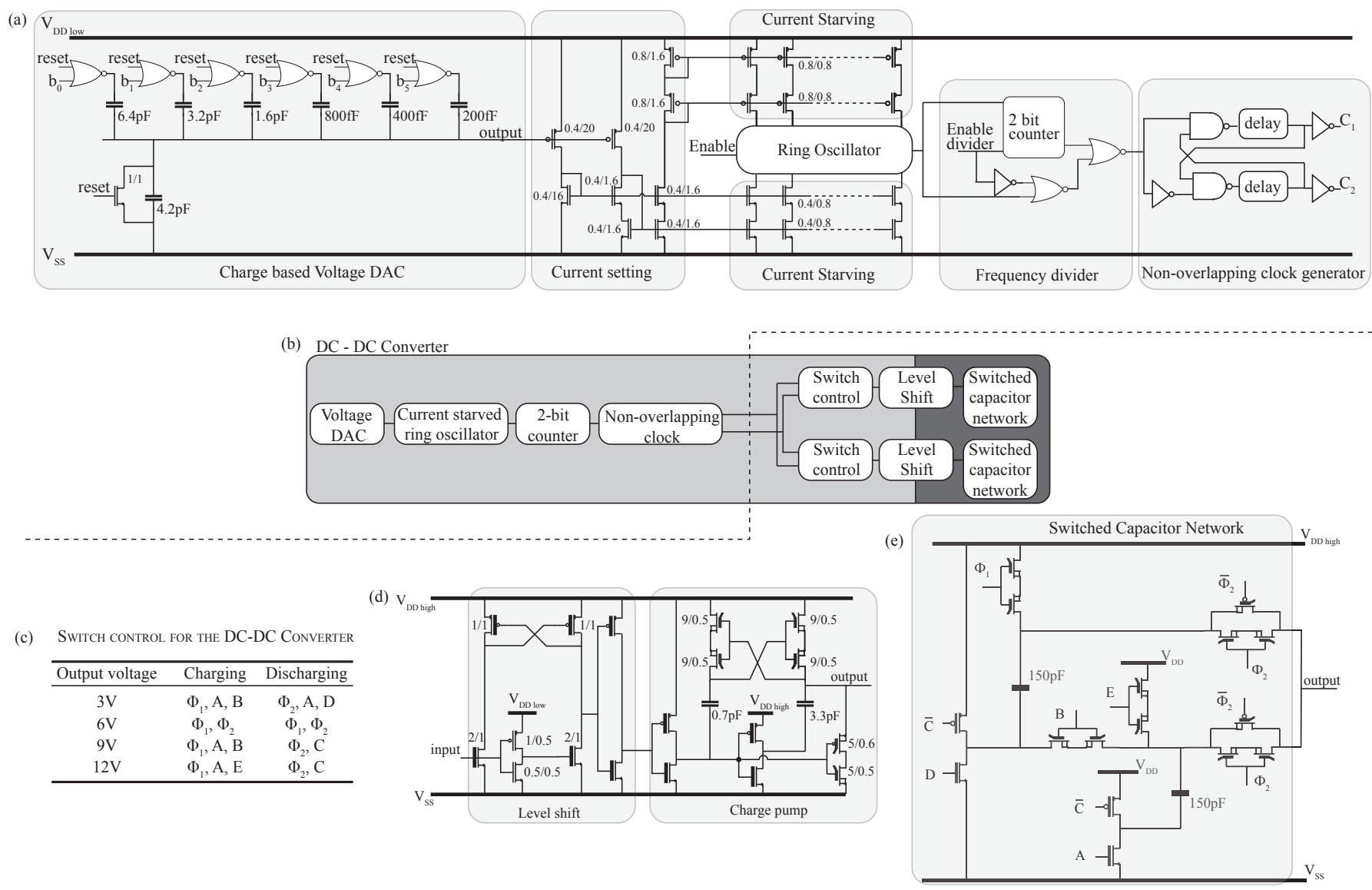

Fig. 8. The DC-DC converter. (a) Circuit schematic of the digitally controlled oscillator comprising of a voltage DAC, a current starved ring oscillator, a frequency divider and a non-overlapping clock generator. (b) Block diagram of the DC-DC converter. (c) Switching combinations for the transistors in the Switched Capacitor network. (d) Level shift and charge pump for driving high voltage transistors from low voltage logic. Mid-voltage transistors are shown with thick gates and high voltage transistors are shown with thick asymmetric gates. PMOS dimensions are $2 / 1 \mu \mathrm{m}$ and NMOS dimensions are $1 / 1 \mu \mathrm{m}$ unless otherwise stated. (e) Circuit schematic showing the switched capacitor network as implemented. All transistors are minimum length, wide devices (width $\gg$ length).

simulations).

At this point the DC-DC converter splits into 2 identical component chains consisting of: a logic block that determines the switches to activate; a level shifting block to enable the $1.8 \mathrm{~V}$ logic signals to drive high voltage transistors; and a switched capacitor network which performs the voltage conversion. Each of these component chains is supplied with both $C_{1}$ and $C_{2}$, but the input pins for the clocks are reversed in one of the chains, causing the two chains to operate in antiphase (i.e. when one chain is charging its switched capacitor network the other is discharging). The level shift circuit used (see Fig. 8d) is a combination between a standard level shift circuit and a clock driver (charge pump) [29], enabling high voltage switches to be driven at approximately twice the supply voltage.

The principle of operation of each of the switched capacitor networks is illustrated in Fig. 9. This shows how each of the networks charge and discharge to each of the output voltages (i.e. $3 \mathrm{~V}, 6 \mathrm{~V}, 9 \mathrm{~V}, 12 \mathrm{~V}$ ). During charging, the capacitors can be connected either in series as a potential divider (Figs 9(b) and (d)) or in parallel (Fig 9(f)) charging the capacitors to $3 \mathrm{~V}$ or $6 \mathrm{~V}$ respectively. During the discharge phase the bottom plate of the capacitor can either be connected to ground, providing an output of $3 \mathrm{~V}$ or to the supply, giving an output of $9 \mathrm{~V}$ or $12 \mathrm{~V}$. As $6 \mathrm{~V}$ is the supply voltage, it is most efficient to simply connect the output to the supply for this voltage. Again it should be noted that due to the clock arrangement when one switched capacitor network is charging the other is discharging, as this greatly reduces the voltage ripple on the output.

The actual implementation of the switched capacitor network is shown in Fig. 8e. Back to back (source to source) high voltage NMOS transistors were used so as to comply with design rules. The operation of the switches (i.e. selection) is shown in Fig 8c.

For the complete DC-DC converter, a total on chip capacitance of $900 \mathrm{pF}$ (using Dual Metal Insulator Metal capacitors) is used for the two switched capacitor networks. This consists of $150 \mathrm{pF}$ for each of the 2 capacitors in each network and a $300 \mathrm{pF}$ load capacitance to limit the output ripple.

\section{Current Sink}

The design of the current sink is shown in Fig. 10. The first stage, consisting of a current reference, produces a stable 


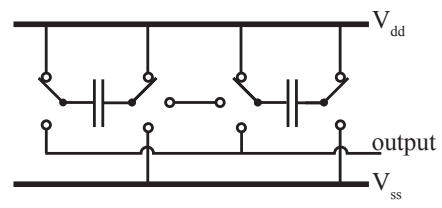

(a) Diagrammatic representation of the switched capacitor network

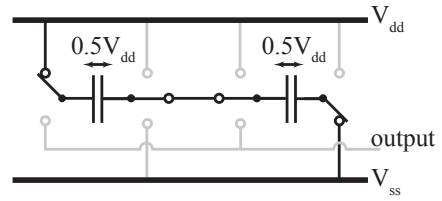

(b) $3 \mathrm{~V}$ charging

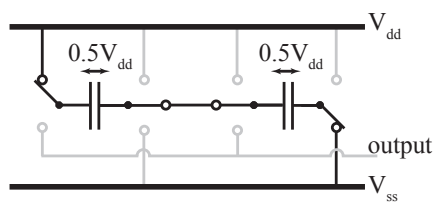

(d) $9 \mathrm{~V}$ charging

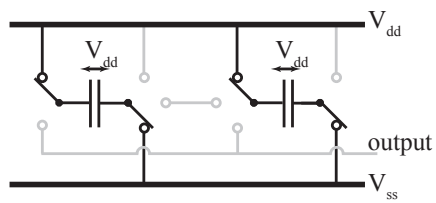

(f) $12 \mathrm{~V}$ charging

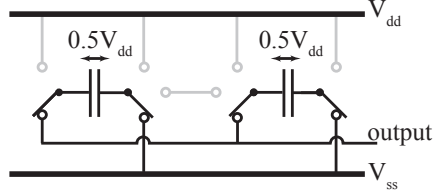

(c) $3 \mathrm{~V}$ discharging

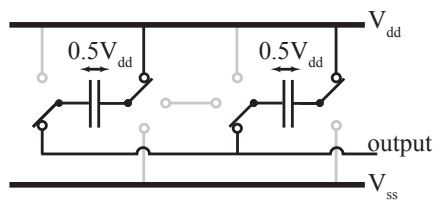

(e) $9 \mathrm{~V}$ discharging

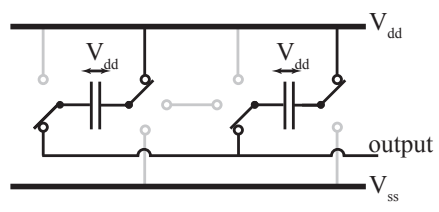

(g) $12 \mathrm{~V}$ discharging

Fig. 9. Charging and discharging cycles of the DC-DC converter. Not shown is the $6 \mathrm{~V}$ output where the supply is shorted to the output.

1.6 $\mu \mathrm{A}$ current which is mirrored into a 6-bit binary-weighted current-DAC. PMOS transistors $\left(S_{1}-S_{6}\right)$ act as switches controlling which branches of the DAC are active and therefore the output of the DAC stage. Due to the binary weighting the gain of the DAC stage is selectable between 0 and $3 \frac{15}{16}$ and as such the output current is controllable in steps of $0.1 \mu \mathrm{A}$ up to $6.3 \mu \mathrm{A}$.

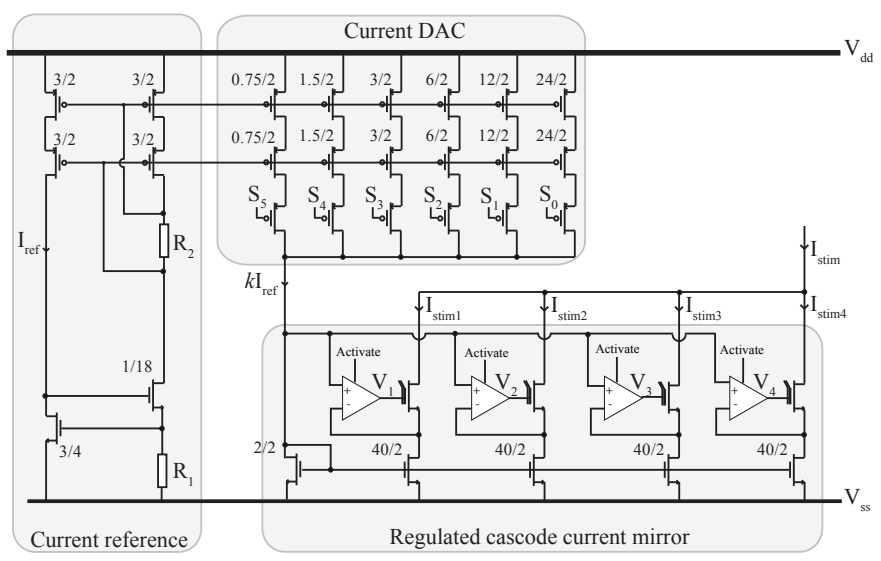

Fig. 10. Circuit schematic of the current reference, DAC and regulated cascode current mirror.

The current generated by the DAC then flows into a regulated cascode current mirror. This mirror is set up with each branch having a W/L ratio 20 times that of the branch

being mirrored so that up to 80 times the DAC output current can flow through the combined sink. A folded cascode opamp driving a high voltage thin-oxide transistor $\left(V_{G S}(\max )=\right.$ $1.8 \mathrm{~V}$ ) is used to regulate the $V_{D S}$ of each of the mirroring transistors [30], [31]. Each op-amp can be individually deactivated, thereby turning off the respective cascode device. During the cathodic stimulation phase all four op-amps are active providing the full factor of 80 gain, however, during the anodic stimulation phase only one of the op-amps is used and as such the gain drops to a factor of 20. This provides the $4: 1$ ratio between the cathodic and anodic phases. The current sink is capable of sinking between $2 \mu \mathrm{A}$ and $504 \mu \mathrm{A}$.

The novel charge balancing approach discussed in Section III-B can now be examined with this system. Looking at Fig. 10, in the cathodic phase the total charge injected $\left(Q_{i}\right)$ into the electrodes will be:

$$
Q_{i}=\left(I_{\text {stim } 1}+I_{\text {stim } 2}+I_{\text {stim } 3}+I_{\text {stim } 4}\right) \times T
$$

where $T$ is the cathodic phase duration. In the subsequent anodic phase, one of the regulated cascodes will be active for 4 times the duration and the charge removed $\left(Q_{r}\right)$ from the electrodes will be (for example):

$$
Q_{r}=I_{\text {stim } 2} \times 4 \times T
$$

The 4 cascoded transistors were matched carefully during layout so this will provide a certain level of charge balancing, but accurate charge balancing can be achieved if the op-amp activated in the anodic phase is changed sequentially for each stimulation, i.e. interleaved. This is because over a series of 4 stimulations each of the mirrors will have been active for the same amount of time anodically as they were cathodically and as such errors due to process variation and mismatch will cancel. The controller has a 2-bit counter for each channel that increments after each anodic stimulation so as to ensure that each op-amp is used in turn.

As mentioned in Section IV-B, the outputs from these opamps are monitored to determine when the cascode transistors are leaving saturation and therefore when to trigger an increase in the DC-DC converter output. The monitoring is achieved using a 2-stage comparator with $100 \mathrm{mV}$ of hysteresis - one terminal is connected to the op-amp output and the other connected to a reference voltage (approximate value $1.4 \mathrm{~V}$ ).

\section{H-bridge}

The H-bridge block consists of an array of 8 H-bridges (one per channel) arranged in parallel and level shifters to drive the high voltage transistors that are used for the switches (in a transmission gate arrangement). The level shift circuit is identical to the one used in the DC-DC converter. The parallel H-bridge arrangement enables a single current sink to selectively pull current through any of the 8 channels, however, this does mean that only a single channel can be active at any moment in time. It is envisaged that the end application will target electrode sites at close proximity and therefore a Continuous Interleave Strategy (CIS) [32], [33] will be used to avoid any cross-stimulation. 


\section{E. Core Supply DC-DC converter}

To operate the system from a single $6 \mathrm{~V}$ supply, a second, auxiliary DC-DC converter has been included to generate the lower voltage supply to the system core. This has been implemented as a serial-parallel switched capacitor converter [34], but utilises the same 2 phase concept as the core DC-DC converter to reduce voltage ripple. The $6 \mathrm{~V}$ (peak to peak) clock for this converter is supplied by an external input and is split into 2 phases using the same non-overlapping clock method as used for the core DC-DC converter.

\section{F. ESD and pad ring}

The combination of high voltage inputs and outputs, and low voltage logic signals requires a split approach to ESD protection and the pad ring. Fig. 11 shows a photograph of the manufactured chip die. Along the left hand side and partway along the top of the die, the high voltage I/O cells can be seen and these are protected with a $20 \mathrm{~V}$ clamp device provided in the foundry models. Along the top-right and right hand side of the die are the low voltage digital and analogue pads for external interfacing and core test. These are standard LV I/O cells protected by a $1.8 \mathrm{~V}$ clamp.

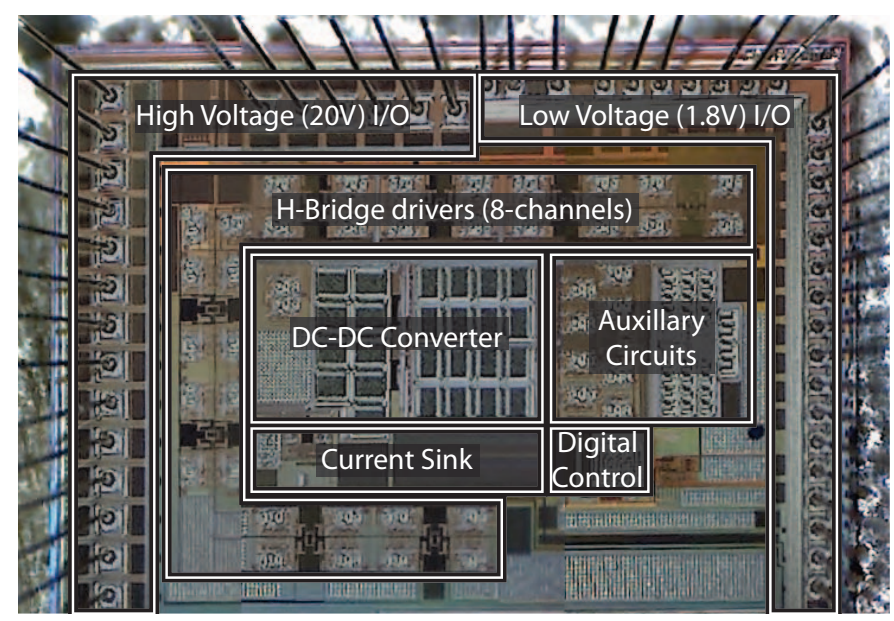

Fig. 11. Microphotograph of the neural stimulator, measuring $2 \mathrm{~mm} \times$ $2.7 \mathrm{~mm}$.

\section{EXPERIMENTAL RESULTS}

The system has been validated through a series of tests including design simulation, bench-top chip measurements and ex-vivo lab experiments on a peripheral nerve.

\section{A. Simulated Results}

The circuit was initially simulated using foundry-supplied PSP models with the Cadence Ultrasim and Spectre simulators. Transient simulations (shown in Fig. 12) for the neural stimulator show that it should be capable of achieving a voltage output of approximately $11.5 \mathrm{~V}$, although between $0.3 \mathrm{~V}$ (at minimum current) and $0.7 \mathrm{~V}$ (at maximum current) of this is required as voltage headroom to keep the current sink transistors in saturation. These results additionally show that despite $100 \mathrm{mV}$ ripple on the DC-DC converter output, the resulting current sink ripple was typically $<2 \mu \mathrm{A}$ (at the maximum current of $504 \mu \mathrm{A}$ ).
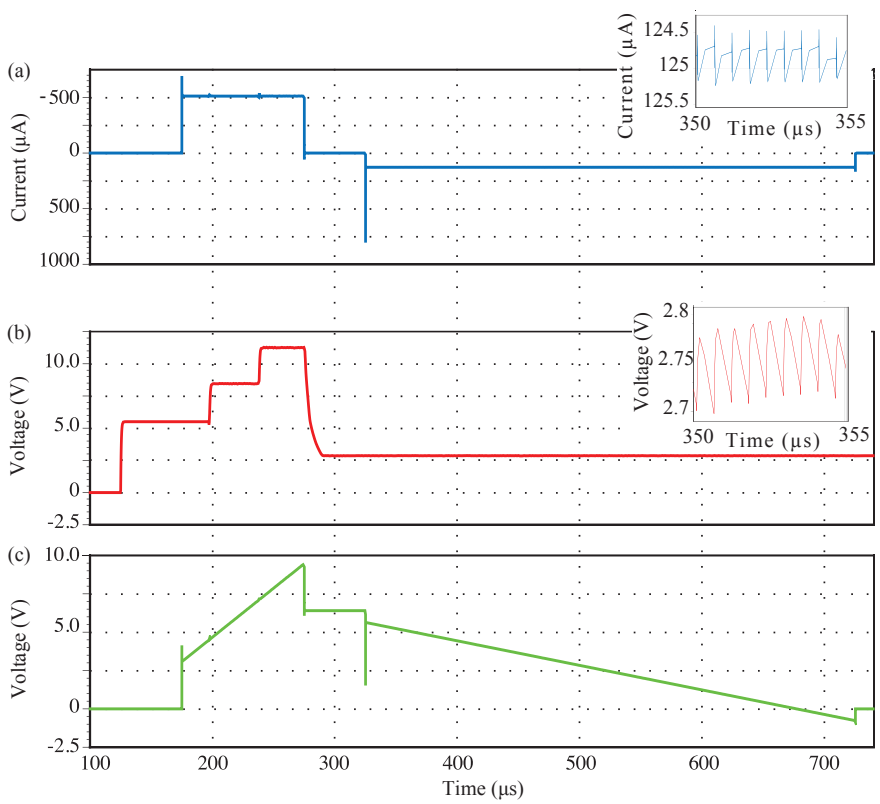

Fig. 12. Transient analysis of the neural stimulator for a full-scale stimulus (504 $\mu \mathrm{A}$ ) showing: (a) stimulation (electrode) current, (b) voltage output of the DC-DC converter, (c) voltage across the electrode model. Insets show close ups of the ripple.

The DC-DC converter efficiency was evaluated under a variety of ideal current loads (with switching frequency tuned to the load) and the results are shown in Fig. 13. These results show that the conversion efficiency is above $80 \%$ for $9 \mathrm{~V}$ and $12 \mathrm{~V}$ outputs across much of the load range. However, the efficiency is worse for light loads and across the entire range for the $3 \mathrm{~V}$ output. This is because the clock, level shift and charge pump overheads make up a significantly higher proportion of the total power consumption at these lower outputs.

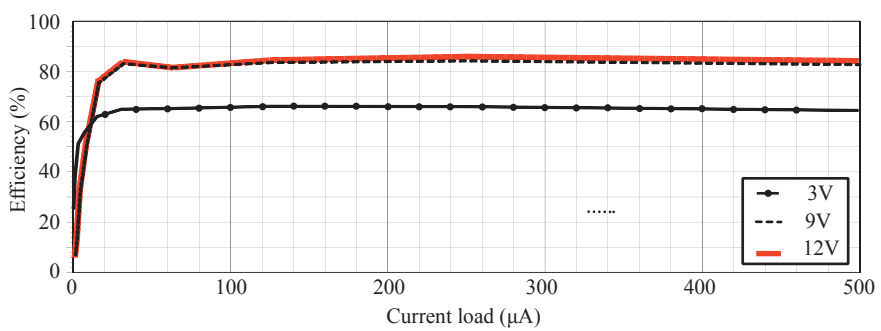

Fig. 13. Simulated DC converter efficiency for different target stimuli (i.e. output current) for $3 \mathrm{~V}, 9 \mathrm{~V}$ and $12 \mathrm{~V}$ compliances.

Monte-Carlo simulations were performed with a fixed DC voltage source to ensure that the proposed charge balance approach was robust to process variation and mismatch (using foundry supplied models of the process). Fig. 14 shows simulated charge imbalance after 1 and 4 stimulation cycles at maximum current. The results show that although there is still some error in charge balance, the likely error is reduced from hundreds of pico-coulombs (for a single pulse) to a few tens 
of pico-coulombs over 4 stimulations. Possible causes of the fixed offset of approximately $-26 \mathrm{pC}$ include charge injection and an inaccuracy in the timing of the signals controlling the stimulation.

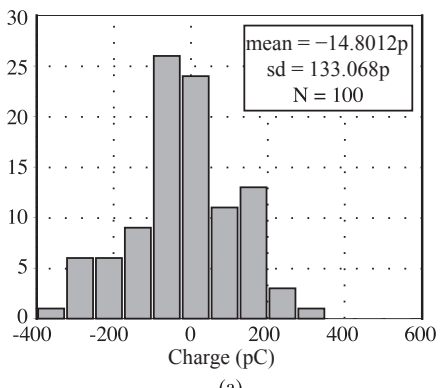

(a)

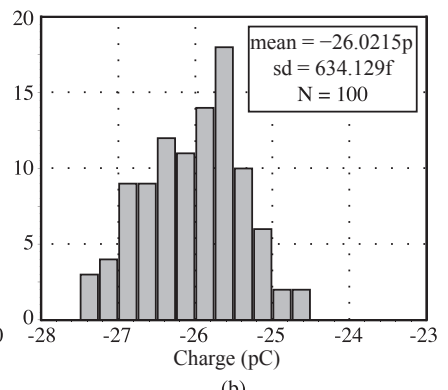

(b)
Fig. 14. Monte Carlo simulations of charge remaining on the electrodes after: (a) single stimulation pulse, and (b) a series of 4 stimulations at maximum stimulation current.

\section{B. Measured results}

The fabricated chips were bonded in a JLCC84 package and mounted on a custom designed PCB test platform. Power was supplied from a Keithley 2602 source meter, the $40 \mathrm{kHz}$ clock was supplied from a function generator, and, for testing purposes, the digital inputs were simply controlled from DIL switches. A lumped model for the electrode was used (consisting of a $6.8 \mathrm{k} \Omega$ resistor in series with 2 parallel ceramic capacitors totalling $6.9 \mathrm{nF}$ ) to approximate our target electrode impedance. Initial investigations focused on verifying that the various subsystems of the chips were working as expected. This was achieved by observing the currents and voltages output by the chip during stimulation cycles. A selection of the measured waveforms are presented in Fig. 15 and in comparison with those presented in Fig. 12 show that the chip is operating as expected, in particular that the maximum voltage output closely matches the $11.5 \mathrm{~V}$ expected. Figs. 15(a) and (c) involved measuring voltage drops across a resistor and this is likely a major contributor to the noise shown on these signals.

Once the functional verification had been completed, performance measurements were undertaken. The first to be measured was the charge balancing performance. This was tested by driving the stimulation current into the charge measuring terminals of a Keithley 6517 a electrometer, which functionally acts as op-amp based charge integrator. The cumulative charge imbalance over 1000 stimulations was measured for a range of stimulation currents and the average charge imbalance is shown in Fig. 16(a) and the percentage error is shown in Fig. 16(b). These results indicate good charge balancing with a charge delivery error of between $0.05 \%$ to $0.15 \%$ across nearly the entire stimulation range with only the lowest stimulation level deviating from this.

The quiescent power consumption of the chips was measured as approximately $185 \mu \mathrm{W}$ using an Agilent 6705b (averaging out as $23.2 \mu \mathrm{W}$ per channel). The dynamic power consumption (during stimulation) of the fabricated chips was measured by connecting a $1.2 \mathrm{k} \Omega$ resistor in series with the

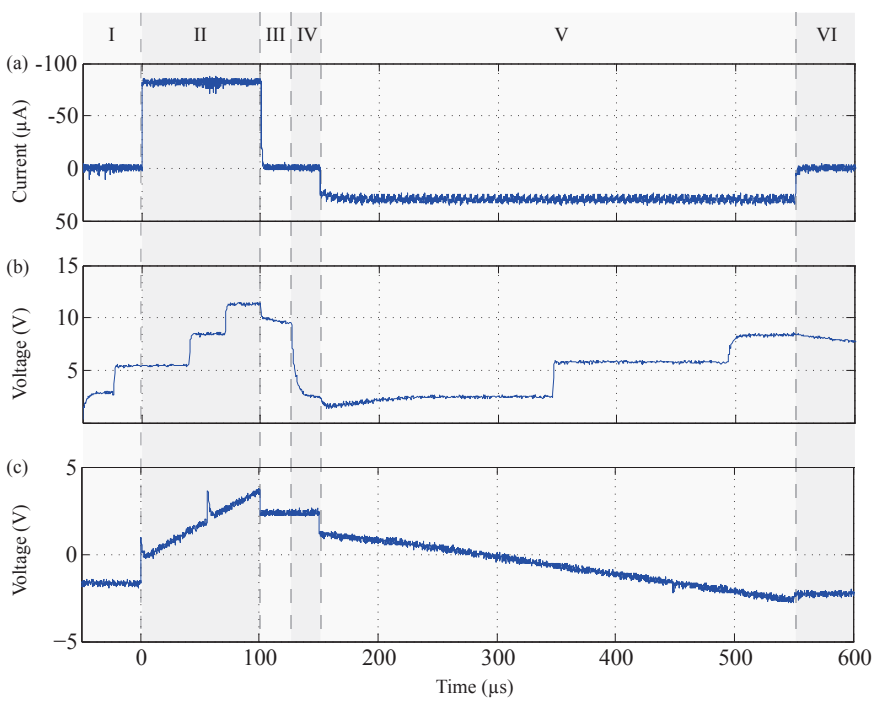

Fig. 15. Sample results of the neural stimulator. Shown are: (a) the current through the lumped electrode model, (b) the output voltage of the DC-DC converter, (c) the electrode voltage during stimulation. Phases I through to VI match the states listed in Table I starting with "Initialise Cathodic".
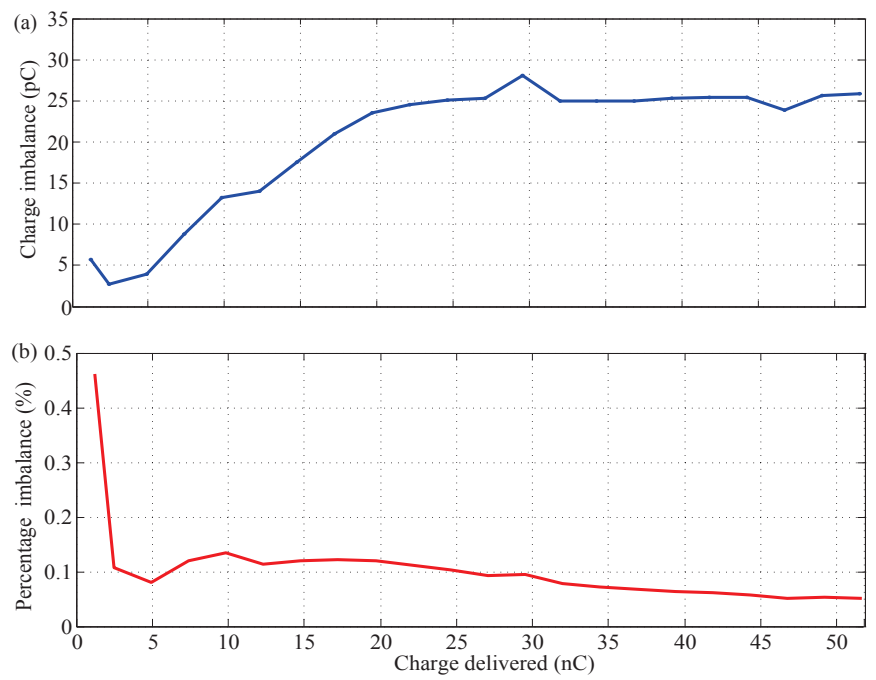

Fig. 16. Charge imbalance results. Shown are (a) the cumulative charge imbalance averaged over 1000 stimulations vs charge injected per stimulation; (b) the charge imbalance as a percentage of charge injected.

$6 \mathrm{~V}$ power supply and monitoring the voltage drop across it on an oscilloscope. This allowed the current, and therefore the power consumed, during a stimulation to be estimated. As proposed in [19] and [23], power savings achieved by this proposed system will be evaluated by comparing with power consumption in the front end of a baseline fixed supply current stimulator that has similar voltage compliance (in our case $11 \mathrm{~V}$ ) and a low voltage headroom. Power consumption estimates for the whole system were gathered for a range of stimulation currents on an electrode $(6.8 \mathrm{k} \Omega$ and $6.9 \mathrm{nF})$ and are shown in Fig. 17(a) alongside simulated and baseline results. The percentage energy reduction that this stimulator provides compared to a standard current mode stimulator is shown in Fig. 17(b). 

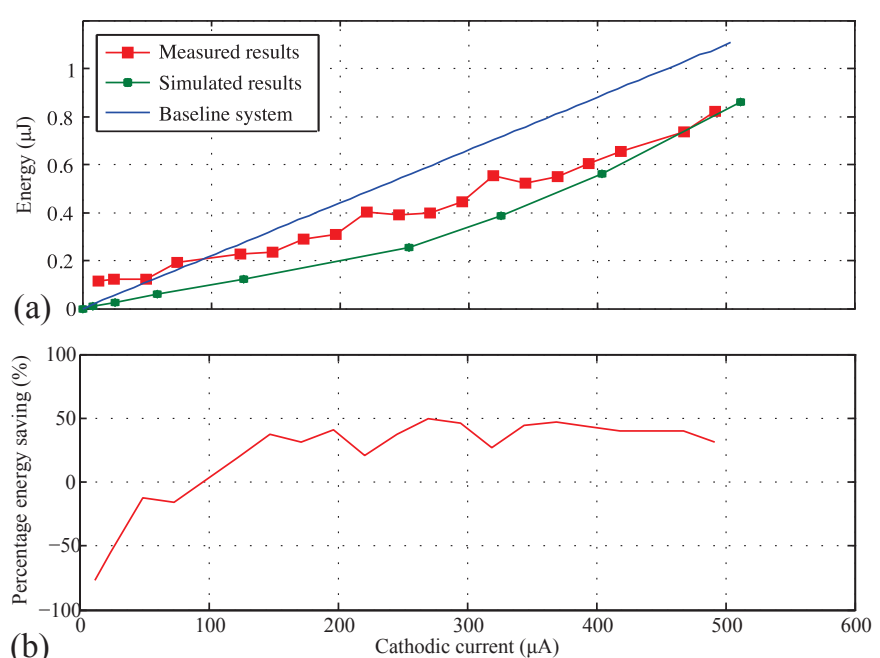

(b)

Fig. 17. Energy per stimulation. Shown are (a) how energy consumption (per stimulation cycle) varies with stimulation current; (b) percentage energy saving by the proposed system.

\section{Ex-Vivo Results}

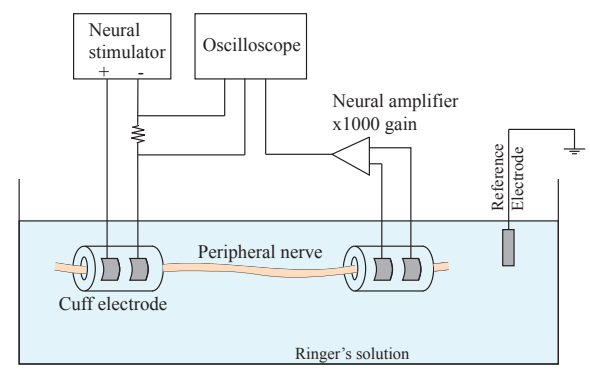

Fig. 18. Experimental setup for the ex-vivo test.

Once the various laboratory tests had been performed to characterise the chip's performance, the core system functionality was validated ex-vivo by stimulating a peripheral nerve. The experimental setup is outlined in Fig. 18. The peripheral nerve was a carefully extracted sciatic nerve (from a dissected African clawed frog - Xenopus laevis) of approximately $10 \mathrm{~cm}$ length, that had been tied at both ends and immersed in amphibian Ringer's solution [35]. A cuff electrode was placed around one end of the nerve and connected to the stimulator. The measured cuff impedance was $3.01 \mathrm{k} \Omega$ and the stimulator was set up to repeatedly deliver and recover $20 \mathrm{nC}$ of charge $(200 \mu \mathrm{A})$ at a rate of 10 stimulations per second. Induced action potentials were measured at the other end of the nerve fascicle and a sample of the recorded data is shown in Fig. 19. The results clearly show that the chip successfully stimulated the nerve fascicle, and an enlarged action potential shows: the stimulation artefacts (I), the cumulative action potential of the fast conducting (A- $\alpha$ ) fibres (II), and the cumulative action potential of the slower conducting (A- $\beta$ ) fibres (III) [36].

\section{Discussion AND Future Work}

This paper has presented a neural stimulator that implements a novel approach to charge balancing - allowing asymmetric
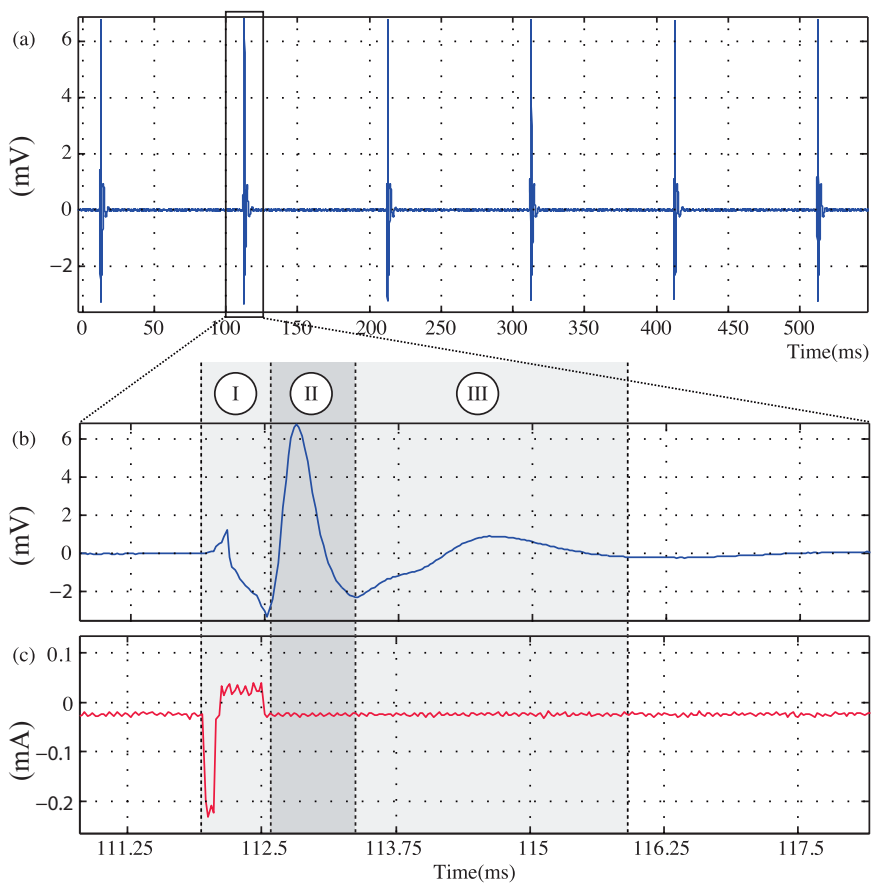

Fig. 19. ex-vivo measured response to stimulation. Shown are: (a) action potentials generated in a peripheral nerve with the neural stimulator, (b) an enlargement of a single stimulated action potential, (c) the measured current stimulus that initiated the action potential.

stimulation patterns to be used - and uses a fully integrated switched capacitor DC-DC converter to reduce system power consumption. Table II compares this work to other designs that achieve high energy-efficiency compared to basic current mode stimulators.

Our work has demonstrated that dynamic voltage scaling using switched capacitor DC-DC conversion can be employed on current-mode stimulation to achieve power savings of up to $50 \%$. The output currents (as seen in Fig. 15 (d)) are stable and show that the DC-DC converter has minimal impact on current stimulus output. The voltage ripple on the output of the converter does cause some current ripple through the electrodes but in simulations this is less than $2 \mu \mathrm{A}$ (peak-to-peak) at the maximum stimulation current. Furthermore, this is at the DC-DC converter clock frequency (i.e. up to $3.3 \mathrm{MHz}$ ) and is thus unlikely to have a physiological impact on stimulation. Power reduction was not achieved at low stimulation currents, likely as a result of the quiescent system power consumption and overheads in operating the DC-DC converter. However, actual power waste in this region is expected to be reasonably small due to the low current levels involved and, assuming the stimulation patterns contain a mixture of high and low stimulus currents, it is likely these power losses will be more than compensated for by the power savings at higher power levels. There are also a number of possible mitigation strategies to avoid power loss at low current levels (e.g. modifying the system to simply use the $6 \mathrm{~V}$ supply at low load currents), however, these incur additional complexity. Unless the low load efficiency of the converter can be significantly improved, it seems unlikely that stimulators operating at currents below $100 \mu \mathrm{A}$ will benefit from DC-DC converter integration. 
TABLE II

COMPARISON WITH OTHER LOW-POWER NEURAL STIMULATOR DESIGNS.

\begin{tabular}{lcccc}
\hline & S. K. Arfin and & S. K. Kelly and & J.-J. Sit and & This work \\
& R. Sarpeskhar [23] & J. L. Wyatt [19] & R. Sarpeskhar [26] & \\
\hline Technology & $0.35 \mu \mathrm{m} \mathrm{CMOS}$ & $1.5 \mu \mathrm{m} \mathrm{CMOS}$ & $0.7 \mu \mathrm{m} \mathrm{HV} \mathrm{CMOS}$ & $0.18 \mu \mathrm{m}$ HV CMOS \\
Dynamic range & $0-450 \mu \mathrm{A}$ & $0-136 \mu \mathrm{A}$ & $0-1 \mathrm{~mA}$ & $0-504 \mu \mathrm{A}$ \\
Voltage compliance & $3 \mathrm{~V}$ & $1.75 \mathrm{~V}$ & $15 \mathrm{~V}$ & $11.5 \mathrm{~V}$ \\
Stimulus control & Current-mode & Voltage-mode & Current-mode & Current-mode \\
Power savings* & $\leq 62 \%$ & $\leq 66 \%$ & n/A & $\leq 50 \%$ \\
Ext. components & Yes & Yes & No & No \\
Charge mismatch & $\mathrm{n} / \mathrm{A}$ & $\mathrm{n} / \mathrm{A}$ & $\leq 0.4 \%$ & $\leq 0.45 \%$ \\
Channels & 1 & 15 & 16 & 8 \\
\hline
\end{tabular}

*Power savings relative to a typical current controlled stimulator

Charge balanced stimulation pulses are important for patient safety and preventing electrode degeneration. DC current flows of $100 \mathrm{nA}$ across an electrode have been correlated with tissue damage in animal models [8] and industry targets a DC error of $<25 \mathrm{nA}$ [26]. The stimulator proposed here is capable of stimulating at over 1500 pulses per second, but will in practice only be used to deliver up to 80 stimulations per second per channel (based on maximum observed firing rates of human proprioceptors [37], [38]). Each stimulation delivers up to $50 \mathrm{nC}$ of charge and therefore this stimulator injects and extracts up to $4 \mu \mathrm{A}$ from the tissue per channel. Measured results show the proposed system delivered charge imbalances of up to $5 \%$ (equivalent to $200 \mathrm{nA}$ ) for a single stimulation and a worst case charge imbalance of $0.46 \%(18 \mathrm{nA})$ for a series of stimulations, with typical charge balancing performance in the $0.05 \%-0.15 \%$ range. The proposed interleaving of current sinks therefore enables asymmetric waveforms while keeping charge balancing performance in the safe operating region.

\section{A. Future Work}

The stimulator presented here is a proof of principle prototype; planned alterations for a future iteration include increasing the number of channels to 16 and reducing the number of bond pads (by removing testing I/O pads and utilising serial rather than parallel data transmission). There are also a number of areas identified for further investigation including:

- The power consumption and issues associated with integrating a voltage regulator to reduce ripple.

- Alternate DC-DC converter control strategies to improve low current stimulation efficiency.

- More comprehensive in vitro testing, including using electrodes of various impedances, to better determine real world efficiency and to investigate the increased current ripple observed in Fig. 18.

- And finally a proposed system redesign to enable the system stimulation voltage $\left(V_{\text {stim }}\right)$ to be set to $0 \mathrm{~V}$ (ground) in the anodic phase - allowing the energy stored in the electrode capacitance to be more efficiently recovered.

\section{ACKNOWLEDGMENT}

This work was supported by the UK Engineering and Physical Sciences Research Council (DTA award and Grant ref: EP/I000569/1). The authors would like to thank Dr Amir
Eftekhar and Dr Jakub Trzebinski for their assistance with the ex-vivo experiments.

\section{REFERENCES}

[1] T. G. Constandinou, J. Georgiou, and C. Toumazou, "A neural implant ASIC for the restoration of balance in individuals with vestibular dysfunction," in Circuits and Systems, 2009. ISCAS 2009. IEEE International Symposium on, May 2009, pp. 641-644.

[2] C. Wall et al., "Vestibular prostheses: The engineering and biomedical issues," Journal of Vestibular Research, vol. 12, no. 2, pp. 95-113, 2003.

[3] C. D. C. Santina et al., "Current and future management of bilateral loss of vestibular sensation; an update on the johns hopkins multichannel vestibular prosthesis project," Cochlear Implants International, vol. 11, no. 3, 2010-09-01T00:00:00.

[4] E. Margalit et al., "Retinal prosthesis for the blind," Survey of Ophthalmology, vol. 47, no. 4, pp. 335-356, 2002.

[5] B. S. Wilson and M. F. Dorman, "Cochlear implants: A remarkable past and a brilliant future," Hearing Research, vol. 242, no. 1 - 2, pp. 3-21, 2008.

[6] S. C. Parisier, P. M. Chute, A. L. Popp, and G. D. Suh, "Outcome analysis of cochlear implant reimplantation in children," The Laryngoscope, vol. 111, no. 1, pp. 26-32, 2001.

[7] S. F. Cogan, "Neural stimulation and recording electrodes," Аnпи. Rev. Biomed. Eng., vol. 10, pp. 275-309, 2008.

[8] R. K. Shepherd, "Chronic electrical stimulation of the auditory nerve using non-charge-balanced stimuli," Acta Oto-laryngologica, vol. 119, no. 6, pp. 674-684, 1999.

[9] I. Williams and T. G. Constandinou, "An energy-efficient, dynamic voltage scaling neural stimulator for a proprioceptive prosthesis," in Circuits and Systems (ISCAS), 2012 IEEE International Symposium on, May 2012, pp. 1091-1094.

[10] U. Proske and S. C. Gandevia, "The proprioceptive senses: Their roles in signaling body shape, body position and movement, and muscle force," Physiological Reviews, vol. 92, no. 4, pp. 1651-1697, 2012.

[11] S. C. Gandevia and D. Burke, "Does the nervous system depend on kinesthetic information to control natural limb movements?" Behavioral and Brain Sciences, vol. 15, pp. 614-632, Nov. 1992.

[12] L. B. Rowell and J. T. Shepherd, Handbook of Physiology: Section 12, Exercise: Regulation and Integration of Multiple Systems. Oxford University Press.

[13] A. M. Barrett et al., "Epidemiology, public health burden, and treatment of diabetic peripheral neuropathic pain: A review," Pain Medicine, vol. 8, no. Supplement s2, pp. S50-S62, 2007.

[14] M. J. Young et al., "A multicentre study of the prevalence of diabetic peripheral neuropathy in the united kingdom hospital clinic population," Diabetologia, vol. 36, pp. 150-154, 1993.

[15] S. Adee, "The revolution will be prosthetized," Spectrum, IEEE, vol. 46, no. 1, pp. 44-48, Jan. 2009.

[16] T. A. Kuiken et al., "Targeted reinnervation for enhanced prosthetic arm function in a woman with a proximal amputation: a case study," The Lancet, vol. 369, no. 9559, pp. 371-380, 2007.

[17] T. Kuiken et al., "Targeted muscle reinnervation for real-time myoelectric control of multifunction artificial arms," JAMA: The Journal of the American Medical Association, vol. 301, no. 6, pp. 619-628, 2009.

[18] A. Blank, A. M. Okamura, and K. J. Kuchenbecker, "Identifying the role of proprioception in upper-limb prosthesis control: Studies on targeted motion," ACM Transactions on Applied Perception, vol. 7, no. 3, 2010. 
[19] S. K. Kelly and J. L. Wyatt, "A power-efficient neural tissue stimulator with energy recovery," Biomedical Circuits and Systems, IEEE Transactions on, vol. 5, no. 1, pp. 20-29, Feb. 2011.

[20] T. Boretius et al., "A transverse intrafascicular multichannel electrode (TIME) to interface with the peripheral nerve," Biosensors and Bioelectronics, vol. 26, no. 1, pp. 62-69, 2010.

[21] K. H. Polasek, H. A. Hoyen, M. W. Keith, and D. J. Tyler, "Human nerve stimulation thresholds and selectivity using a multi-contact nerve cuff electrode," Neural Systems and Rehabilitation Engineering, IEEE Transactions on, vol. 15, no. 1, pp. 76-82, Mar. 2007.

[22] M. A. Schiefer et al., "Selective stimulation of the human femoral nerve with a flat interface nerve electrode," Journal of Neural Engineering, vol. 7, no. 2, pp. 195-204.

[23] S. K. Arfin and R. Sarpeshkar, "An energy-efficient, adiabatic electrode stimulator with inductive energy recycling and feedback current regulation," Biomedical Circuits and Systems, IEEE Transactions on, vol. 6, no. 1, pp. 1-14, Feb. 2012.

[24] S. Ethier, M. Sawan, E. M. Aboulhamid, and M. El-Gamal, "A 9v fully integrated cmos electrode driver for high-impedance microstimulation," in Circuits and Systems, 2009. MWSCAS 09. 52nd IEEE International Midwest Symposium on, Aug. 2009, pp. 192-195.

[25] V. Kursun and E. G. Friedman, Multi-voltage CMOS circuit design. John Wiley \& Sons, Ltd.

[26] J.-J. Sit and R. Sarpeshkar, "A low-power blocking-capacitor-free charge-balanced electrode-stimulator chip with less than $6 \mathrm{nA}$ DC error for 1-mA full-scale stimulation," Biomedical Circuits and Systems, IEEE Transactions on, vol. 1, no. 3, pp. 172-183, Sep. 2007.

[27] K. Sooksood, T. Stieglitz, and M. Ortmanns, "An active approach for charge balancing in functional electrical stimulation," Biomedical Circuits and Systems, IEEE Transactions on, vol. 4, no. 3, pp. 162 170 , june 2010.

[28] O. Macherey et al., "Asymmetric Pulses in Cochlear Implants: Effects of Pulse Shape, Polarity, and Rate," JARO - Journal of the Association for Research in Otolaryngology, no. 3, pp. 253-266.

[29] R. J. Baker, CMOS: Circuit Design, Layout and Simulation. John Wiley $\&$ Sons, Inc.

[30] M. Ghovanloo and K. Najafi, "A compact large voltage-compliance high output-impedance programmable current source for implantable microstimulators," Biomedical Engineering, IEEE Transactions on, vol. 52, no. 1, pp. 97-105, Jan. 2005.

[31] M. Azin and P. Mohseni, "A high-output-impedance current microstimulator for anatomical rewiring of cortical circuitry," in Circuits and Systems, 2008. ISCAS 2008. IEEE International Symposium on, May 2008, pp. 2502-2505.

[32] B. S. Wilson et al., "Design and evaluation of a continuous interleaved sampling (CIS) processing strategy for multichannel cochlear implants," Journal of Rehabilitation Research, vol. 30, no. 1, pp. 110-116, 1993.

[33] T. G. Constandinou, J. Georgiou, and C. Toumazou, "A partial-currentsteering biphasic stimulation driver for vestibular prostheses," Biomedical Circuits and Systems, IEEE Transactions on, vol. 2, no. 2, pp. 106113, Jun. 2008.

[34] M. S. M. Wens, Design and Implementation of Fully-Integrated Inductive DC-DC Converters in Standard CMOS, Analog Circuits and Signal Processing,, 1st ed. Springer Science and Business Media, 2011.
[35] Z. Mou et al., "A simulation study of the combined thermoelectric extracellular stimulation of the sciatic nerve of the xenopus laevis: The localized transient heat block," Biomedical Engineering, IEEE Transactions on, vol. 59, no. 6, pp. 1758-1769, Jun. 2012.

[36] V. M. Woods, "Stimulus waveforms based on platinum polarization for efficient and selective nerve stimulation," $\mathrm{PhD}$ thesis, Imperial College London, 2011.

[37] L. Jami, "Golgi tendon organs in mammalian skeletal muscle: Functional properties and central actions," Physiological Reviews, vol. 72, no. 3, pp. 623-666, 1992.

[38] B. B. Edin and Å. B. Vallbo, "Dynamic response of human muscle spindle afferents to stretch," Journal of Neurophysiology, vol. 63, no. 6, pp. 1297-1306, 1990

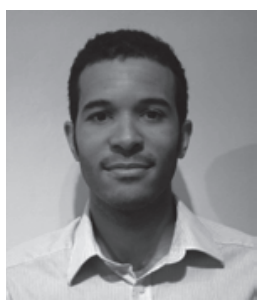

Ian Williams received the M.Eng. degree in electronic engineering from Edinburgh University, UK, in 2004

From 2004 to 2010 he worked in a number of project management and research related roles for the UK Ministry of Defence. He is currently working towards the Ph.D. in Electronic Engineering at Imperial College London, UK. His research interests include brain-machine interfaces and his doctoral research is focused on developing a neural proprioceptive prosthesis for upper limb amputees.

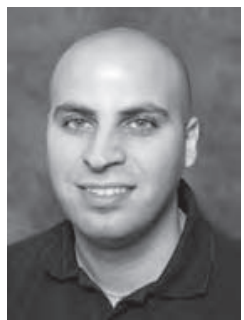

Timothy G. Constandinou (AM'98-M'01-SM'10) received the B.Eng. degree in Electrical and Electronic Engineering in 2001 and the Ph.D. degree in 2005 both from Imperial College London. He then moved to the Institute of Biomedical Engineering (also at Imperial) where he was appointed to Research Officer in Bionics until joining academic faculty in 2010 . He is currently a lecturer within the Department of Electrical \& Electronic Engineering at Imperial College London and is also the deputy director of the Centre for Bio-Inspired Technology. His research utilises integrated circuit and microsystem technologies to address challenges in implantable neural prosthetics, brain-machine interfaces, lab-onchip/wireless capsule endoscope platforms and medical devices in general. $\mathrm{He}$ is a Senior Member of the IEEE, a Fellow of the IET, a Chartered Engineer and Member of the IoP and SPIE. He currently serves on the BioCAS and Sensory Systems technical committees of the IEEE CAS Society and chairs the IET awards and prizes committee. He was the Technical Program CoChair of the 2010 and 2011 IEEE BioCAS Conferences, Technical Program Track Co-Chair (Bioengineering) of the 2012 IEEE ICECS Conference and Technical Program Track Chair (ASICs) of the 2012 BSN Conference. 